\title{
HANDBOOK of GRAPH GRAMMARS and COMPUTING by GRAPH TRANSFORMATION
}




\title{
HANDBOOK OF GRAPH GRAMMARS AND COMPUTING BY GRAPH TRANSFORMATION
}

\author{
Managing Editor: G. Rozenberg, Leiden, The Netherlands \\ Advisory Board: $\quad$ B. Courcelle, Bordeaux, France \\ $\mathrm{H}$. Ehrig, Berlin, Germany \\ G. Engels, Leiden, The Netherlands \\ D. Janssens, Antwerp, Belgium \\ H.-J. Kreowski, Bremen, Germany \\ U. Montanari, Pisa, Italy
}

Vol. 1: Foundations

Forthcoming:

Vol. 2: Specifications and Programming

Vol. 3: Concurrency 
Volume 1

\section{FOUNDATIONS}

\section{HANDBOOK of \\ GRAPH GRAMMARS and COMPUTING by GRAPH TRANSFORMATION}

Edited by

Grzegorz Rozenberg

Leiden University, The Netherlands 
Published by

World Scientific Publishing Co. Pte. Ltd.

P O Box 128, Farrer Road, Singapore 912805

USA office: Suite 1B, 1060 Main Street, River Edge, NJ 07661

UK office: 57 Shelton Street, Covent Garden, London WC2H 9HE

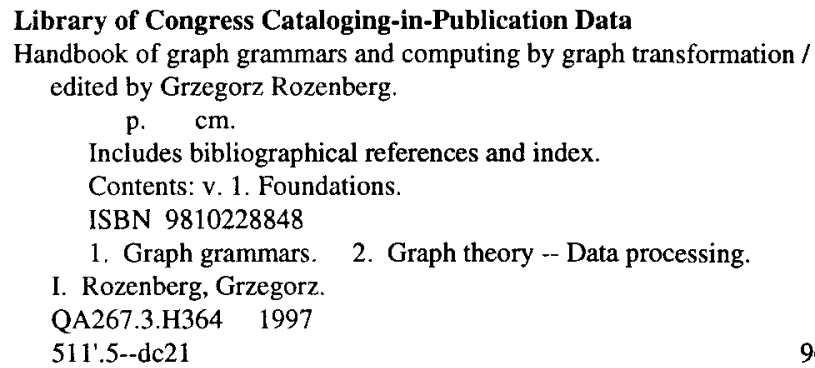

\section{British Library Cataloguing-in-Publication Data}

A catalogue record for this book is available from the British Library.

Copyright $(9) 1997$ by World Scientific Publishing Co. Pte. Ltd.

All rights reserved. This book, or parts thereof, may not be reproduced in any form or by any means, electronic or mechanical, including photocopying, recording or any information storage and retrieval system now known or to be invented, without written permission from the Publisher.

For photocopying of material in this volume, please pay a copying fee through the Copyright Clearance Center, Inc., 222 Rosewood Drive, Danvers, MA 01923, USA. In this case permission to photocopy is not required from the publisher.

This book is printed on acid-free paper.

Printed in Singapore by Uto-Print 


\section{Preface}

Graph grammars originated in the late 60 's, motivated by considerations about pattern recognition, compiler construction, and data type specification. Since then the list of areas which have interacted with the development of graph grammars has grown impressively. Besides the aforementioned areas it includes software specification and development, VLSI layout schemes, database design, modelling of concurrent systems, massively parallel computer architectures, logic programming, computer animation, developmental biology, music composition, visual languages, and many others. Graph grammars are interesting from the theoretical point of view because they are a natural generalization of formal language theory based on strings and the theory of term rewriting based on trees.

The wide applicability of graph grammars is due to the fact that graphs are a natural way of describing complex situations on an intuitive level. Moreover, the graph transformations associated with a graph grammar bring "dynamic behaviour" to such descriptions, because they model the evolution of graphical structures. Therefore graph grammars and graph transformations become attractive as a "programming paradigm" for software and graphical interfaces.

Over the last 25-odd years graph grammars have developed at a steady pace into a theoretically sound and well-motivated research field. In particular, they are now based on solid foundations, presented in this volume. It includes a state-of-the-art presentation of the foundations of all basic approaches to graph grammars and computing by graph transformations.

The two most basic choices for rewriting a graph are node replacement and edge replacement, or, in the more general setting of hypergraphs, hyperedge replacement. In a node replacement graph grammar a node of a given graph is replaced by a new subgraph which is connected to the remainder of the graph by new edges depending on how the node was connected to it. In a hyperedge replacement graph grammar a hyperedge of a given hypergraph is replaced by a new subhypergraph which is glued to the remainder of the hypergraph by fusing (identifying) some nodes of the subhypergraph with some nodes of the remainder of the hypergraph depending on how the hyperedge was glued to it. Chapter 1 surveys the theory of node replacement graph grammars concentrating mainly on "context-free" (or "confluent") node replacement graph grammars, while Chapter 2 surveys the theory of hyperedge replacement graph grammars. Both types of graph grammars naturally generalize the context-free string grammars. 
The gluing of graphs plays also a central role in the algebraic approach to graph transformations, where a subgraph, rather than a node or an edge only, can be replaced by a new subgraph (generalizing in this way arbitrary type-0 Chomsky string grammars). Originally, the rewriting of graphs based on gluing has been formulated by the so-called double pushout in the category of graphs and total graph morphisms. More recently a single pushout in the category of graphs and partial graph morphisms has been used for this purpose. Chapter 3 gives an overview of the double pushout approach, and Chapter 4 gives an overview of the single pushout approach; it also presents a detailed comparison of the two approaches.

Graphs may be considered as logical structures and so one can express formally their properties by logical formulas. Consequently classes of graphs may be described by formulas of appropriate logical languages. Such logical formulas are used as finite devices, comparable to grammars and automata, to specify classes of graphs and to deduce properties of such classes from their logical descriptions. Chapter 5 surveys the relationships between monadic secondorder logic and the context-free graph grammars of Chapters 1 and 2.

The research on graph transformations leads to a careful re-thinking of the formal framework that should be used for the specification of graphs. The theory of 2-structures, a specific relational framework, has turned out to be fruitful for the investigation of graphs especially in their relationship to graph transformations. The "static part" of the theory of 2-structures allows to obtain rather strong decomposition results for graphs, while the "dynamic part" of the theory employs group theory to consider transformations of graphs as encountered in networks. Chapter 6 presents the basic theory of 2-structures.

In order to specify classes of graphs and graph transformations as they occur in various applications (e.g. databases and database manipulations) one often needs quite powerful extensions of the basic mechanisms of graph replacement systems. One such extension is to control the order of application of the graph replacement rules. Chapter 7 presents a basic framework for programmed graph replacement systems based on such a control.

We believe that this volume together with the two forthcoming volumes - on specification and programming, and on concurrency - provide the reader with a rather complete insight into the mathematically challenging area of graph grammars which is well motivated by its many applications to computer science and beyond. 
My thanks go first of all to the graph grammar community - the enthusiastic group of researchers, often with very different backgrounds, spread all over the world - for providing a very stimulating environment for the work on graph grammars. Perhaps the main driving force in this community during the last 7 years was the ESPRIT Basic Research Working Group COMPUGRAPH (Computing by Graph Transformation) in the period March 1989 - February 1992, followed by the ESPRIT Basic Research Working Group COMPUGRAPH II in the period October 1992 - March 1996. The initial planning of the handbook took place within the COMPUGRAPH project, and most of Volume I of the handbook has been written within the COMPUGRAPH period. The European Community is also founding now the TMR network GETGRATS (General Theory of Graph Transformation Systems) for the period from September 1996 to August 1999. We hope to complete volumes II and III of the handbook within the GETGRATS project. The gratitude of the graph grammar community goes to the European Community for the generous support of our research. We are also indebted to $\mathrm{H}$. Ehrig and his Berlin group for managing the COMPUGRAPH and COMPUGRAPH II Working Groups, and to A. Corradini and U. Montanari for their work in preparing the GETGRATS project.

I am very grateful to all the authors of the chapters of this volume for their cooperation, and to the Advisory Board: B. Courcelle, H. Ehrig, G. Engels, D. Janssens, H.-J. Kreowski and U. Montanari, for their valuable advice.

Finally, very special thanks go to Perdita Löhr for her help in transforming all the separate chapters into a homogeneous and readable volume as it is now. Neither she nor myself could foresee how much work it would be - but due to her efforts we could bring the project to a happy end.

\author{
G. Rozenberg \\ Managing Editor \\ Leiden, 1996
}


This page is intentionally left blank 


\section{Contents}

1 Node Replacement Graph Grammars 1

(J. Engelfriet, G. Rozenberg) 1

1.1 Introduction . . . . . . . . . . . . . . 3

1.2 From NLC to edNCE . . . . . . . . . . . . . . . 4

1.2.1 Node replacement and the NLC methodology . . . . . . 4

1.2.2 Extensions and variations: the edNCE grammar . . . 9

1.2.3 Graph replacement grammars . . . . . . . . . . . 14

1.2.4 Bibliographical comments . . . . . . . . . . . . . 15

1.3 Node replacement grammars with Neighbourhood Controlled Embedding . . . . . . . . . . . . . . . 16

1.3.1 Formal definition of edNCE graph grammars . . . . 16

1.3.2 Leftmost derivations and derivation trees . . . . . . 38

Leftmost derivations . . . . . . . . . . . 38

Derivation trees . . . . . . . . . . . . . 43

1.3.3 Subclasses . . . . . . . . . . . . . . . . 55

1.3 .4 Normal forms . . . . . . . . . . . . . . . 61

1.4 Characterizations . . . . . . . . . . . . . 68

1.4.1 Regular path characterization . . . . . . . . . . 68

1.4.2 Logical characterization . . . . . . . . . . . . . 72

1.4.3 Handle replacement . . . . . . . . . . . . . . . . 79

1.4.4 Graph expressions . . . . . . . . . . . . . . . 81

1.5 Recognition . . . . . . . . . . . . . . 82

References ........................... 88

2 Hyperedge Replacement Graph Grammars 95

(F. Drewes, H.-J. Kreowski, A. Habel) 95

2.1 Introduction . . . . . . . . . . . . . . . . . 97

2.2 Hyperedge replacement grammars . . . . . . . . . . . 100

2.2 .1 Hypergraphs . . . . . . . . . . . . . 102

2.2 .2 Hyperedge replacement . . . . . . . . . . . . 104

2.2.3 Hyperedge replacement derivations, grammars, and languages . . . . . . . . . . . . . 105

2.2 .4 Bibliographic notes . . . . . . . . . . . . 109

2.3 A context-freeness lemma . . . . . . . . . . . . . . 111

2.3.1 Context freeness . . . . . . . . . . . . . . . . . 111

2.3.2 Derivation trees. . . . . . . . . . . . . . . . 114

2.3.3 Bibliographic notes . . . . . . . . . . . . 115

2.4 Structural properties . . . . . . . . . . . . . 116

2.4 .1 A fixed-point theorem . . . . . . . . . . 116 
2.4 .2 A pumping lemma . . . . . . . . . . . . 118

2.4 .3 Parikh's theorem . . . . . . . . . . . . . 122

2.4 .4 Bibliographic notes . . . . . . . . . . . 123

2.5 Generative power . . . . . . . . . . . . . . . . . . 124

2.5.1 Graph-generating hyperedge replacement grammars . . 124

2.5.2 String-generating hyperedge replacement grammars . . 125

2.5.3 Further results and bibliographic notes . . . . . . . 130

2.6 Decision problems . . . . . . . . . . . . . . . 132

2.6.1 Compatible properties . . . . . . . . . . . 132

2.6.2 Compatible functions . . . . . . . . . . . . 135

2.6.3 Further results and bibliographic notes . . . . . . 138

2.7 The membership problem . . . . . . . . . . . . . 141

2.7.1 NP-completeness . . . . . . . . . . . . . 141

2.7 .2 Two polynomial algorithms . . . . . . . . . 145

2.7.3 Further results and bibliographic notes . . . . . . . . 154

2.8 Conclusion . . . . . . . . . . . . . . . . 155

References . . . . . . . . . . . . . . . 156

3 Algebraic Approaches to Graph Transformation - Part I: Basic Concepts and Double Pushout Approach 163

(A. Corradini, U. Montanari, F. Rossi, H. Ehrig, R. Heckel, M. Löwe) 163

3.1 Introduction . . . . . . . . . . . . . . . 165

3.2 Overview of the Algebraic Approaches . . . . . . . . . . 168

3.2.1 Graphs, Productions and Derivations . . . . . . . . . 168

3.2 .2 Independence and Parallelism . . . . . . . . . . . . 172

Interleaving . . . . . . . . . . . . . . 172

Explicit Parallelism . . . . . . . . . . . . . . 174

3.2.3 Embedding of Derivations and Derived Productions . . 176

3.2.4 Amalgamation and Distribution . . . . . . . . 178

Amalgamation . . . . . . . . . . . . 178

Distribution . . . . . . . . . . . . . . . 179

3.2.5 Further Problems and Results . . . . . . . . . . . 180

Semantics . . . . . . . . . . . . . . . 180

Control . . . . . . . . . . . . . . . . 181

Structuring . . . . . . . . . . . . . . 182

Analysis . . . . . . . . . . . . . . 182

More general structures . . . . . . . . . . . . . 182

3.3 Graph Transformation Based on the DPO Construction . . . 182

3.4 Independence and Parallelism in the DPO approach . . . . . 191

3.5 Models of Computation in the DPO Approach . . . . . . . 200 
3.5.1 The Concrete and Truly Concurrent Models of Computation . . . . . . . . . . . . . . . . 201

3.5.2 Requirements for capturing representation independence. 208

3.5.3 Towards an equivalence for representation independence. 212

3.5.4 The abstract models of computation for a grammar. . . 217

3.6 Embedding, Amalgamation and Distribution in the

DPO approach . . . . . . . . . . . . . 220

3.6.1 Embedding of Derivations and Derived Productions . , 220

3.6.2 Amalgamation and Distribution . . . . . . . . 224

Amalgamation . . . . . . . . . . . . 224

Distribution . . . . . . . . . . . . . 225

3.7 Conclusion . . . . . . . . . . . . . . . . 228

3.8 Appendix A: On commutativity of coproducts . . . . . . 228

3.9 Appendix B: Proof of main results of Section $3.5 \ldots 232$

References . . . . . . . . . . . . . . . . 240

4 Algebraic Approaches to Graph Transformation - Part II: Single Pushout Approach and Comparison with Double Pushout Approach

(H. Ehrig, R. Heckel, M. Korff, M. Löwe, L. Ribeiro, A. Wagner,

A. Corradini) $\quad 247$

4.1 Introduction . . . . . . . . . . . . . . . . . 249

4.2 Graph Transformation Based on the SPO Construction . . . . 250

4.2.1 Graph Grammars and Derivations in the SPO Approach 250

4.2.2 Historical Roots of the SPO Approach . . . . . . . . 258

4.3 Main Results in the SPO Approach . . . . . . . . . . . . 259

4.3.1 Parallelism . . . . . . . . . . . . . 260

Interleaving . . . . . . . . . . . . 260

Explicit Parallelism . . . . . . . . . . . . . 264

4.3.2 Embedding of Derivations and Derived Productions . . 268

4.3.3 Amalgamation and Distribution ........... 273

4.4 Application Conditions in the SPO Approach . . . . . . . . 278

4.4.1 Negative Application Conditions . . . . . . . . . 278

4.4.2 Independence and Parallelism of Conditional Derivations 282

Interleaving . . . . . . . . . . . . . . 282

Explicit Parallelism . . . . . . . . . . . . . . . 284

4.5 Transformation of More General Structures in the

SPO Approach . . . . . . . . . . . . . . . . 287

4.5.1 Attributed Graphs . . . . . . . . . . . . . . . . 288

4.5.2 Graph Structures and Generalized Graph Structures . . 292 
4.5.3 High-Level Replacement Systems . . . . . . . . . . . 294

4.6 Comparison of DPO and SPO Approach . . . . . . . . . . 296

4.6.1 Graphs, Productions, and Derivations . . . . . . 298

4.6.2 Independence and Parallelism . . . . . . . . . . . 301

Interleaving . . . . . . . . . . . . . 301

Explicit Parallelism . . . . . . . . . . . . 302

4.6.3 Embedding of Derivations and Derived Productions . . 305

4.6.4 Amalgamation and Distribution . . . . . . . 306

4.7 Conclusion . . . . . . . . . . . . . . . . 308

References . . . . . . . . . . . . . . . . . 309

5 The Expression of Graph Properties and Graph Transformations in Monadic Second-Order Logic 313 (B. Courcelle) $\quad \mathbf{3 1 3}$

5.1 Introduction . . . . . . . . . . . . . . . . . 315

5.2 Relational structures and logical languages . . . . . . . . 317

5.2 .1 Structures . . . . . . . . . . . . . . . 317

5.2 .2 First-order logic $\ldots \ldots \ldots \ldots \ldots \ldots$

5.2 .3 Second-order logic . . . . . . . . . . . . . . . . . 319

5.2 .4 Monadic second-order logic . . . . . . . . . . . 320

5.2 .5 Decidability questions . . . . . . . . . . . 322

5.2 .6 Some tools for constructing formulas . . . . . . . . 324

5.2.7 Transitive closure and path properties . . . . . . . 327

5.2.8 Monadic second-order logic without individual variables 331

5.2.9 A worked example: the definition of square grids in MS

$\operatorname{logic} \ldots \ldots \ldots \ldots \ldots \ldots . \ldots \ldots 32$

5.3 Representations of partial orders, graphs and hypergraphs by relational structures . . . . . . . . . . . . . 334

5.3 .1 Partial orders . . . . . . . . . . . . . 334

5.3 .2 Edge set quantifications . . . . . . . . . . 335

5.3 .3 Hypergraphs . . . . . . . . . . . . . . . . . . 339

5.4 The expressive powers of monadic-second order languages . . 340

5.4 .1 Cardinality predicates . . . . . . . . . . . 340

5.4 .2 Linearly ordered structures . . . . . . . . . . . 342

5.4 .3 Finiteness . . . . . . . . . . . . . . . . . 344

5.5 Monadic second-order definable transductions . . . . . . . . 346

5.5.1 Transductions of relational structures . . . . . . . 346

5.5.2 The fundamental property of definable transductions . . 351

5.5.3 Comparisons of representations of partial orders, graphs and hypergraphs by relational structures . . . . . . . 354 
5.6 Equational sets of graphs and hypergraphs . . . . . . . . 356

5.6 .1 Equational sets . . . . . . . . . . . . . 356

5.6.2 Graphs with ports and VR sets of graphs . . . . . . . 362

5.6.3 Hypergraphs with sources and HR sets of hypergraphs . 367

5.7 Inductive computations and recognizability . . . . . . . . 372

5.7.1 Inductive sets of predicates and recognizable sets . . . 372

5.7.2 Inductivity of monadic second-order predicates . . . . 379

5.7.3 Inductively computable functions and a generalization of Parikh's theorem . . . . . . . . . . . . . . 383

5.7.4 Logical characterizations of recognizability . . . . . . . 389

5.8 Forbidden configurations . . . . . . . . . . . . . . . 390

5.8 .1 Minors . . . . . . . . . . . . . . 390

5.8.2 The structure of sets of graphs having decidable monadic theories . . . . . . . . . . . . . 396

References . . . . . . . . . . . . . . . 397

6 2-Structures - A Framework For Decomposition And Transformation Of Graphs

(A. Ehrenfeucht, T. Harju, G. Rozenberg) 401

6.1 Introduction . . . . . . . . . . . . . . 403

6.2 2-Structures and Their Clans . . . . . . . . . . . . . 404

6.2.1 Definition of a 2-structure . . . . . . . . . . . . 404

6.2 .2 Clans . . . . . . . . . . . . . . 407

6.2.3 Basic properties of clans . . . . . . . . . . . 410

6.3 Decompositions of 2 -Structures . . . . . . . . . . . . 411

6.3 .1 Prime clans . . . . . . . . . . . . . . 411

6.3 .2 Quotients .................. . . 412

6.3 .3 Maximal prime clans . . . . . . . . . . . . 416

6.3.4 Special 2-structures ............. . . 418

6.3.5 The clan decomposition theorem . . . . . . . . . 419

6.3.6 The shape of a 2-structure . . . . . . . . . . . . 421

6.3.7 Constructions of clans and prime clans . . . . . . . . . 424

6.3.8 Clans and sibas . . . . . . . . . . . . . . 427

6.4 Primitive 2 -Structures . . . . . . . . . . . . . . . . . 430

6.4 .1 Hereditary properties . . . . . . . . . . . . 430

6.4 .2 Uniformly non-primitive 2 -structures . . . . . . . . 433

6.5 Angular 2-structures and T-structures . . . . . . . . . . . 434

6.5 .1 Angular 2-structures . . . . . . . . . . . . 434

6.5.2 T-structures and texts . . . . . . . . . . 438

6.6 Labeled 2-Structures . . . . . . . . . . . . . . . . . 442 
6.6.1 Definition of a labeled 2-structure . . . . . . . . 442

6.6.2 Substructures, clans and quotients . . . . . . . . . 444

6.7 Dynamic Labeled 2-Structures . . . . . . . . . . . . 447

6.7.1 Motivation . . . . . . . . . . . . . . 447

6.7 .2 Group labeled 2-structures . . . . . . . . . . . . 449

6.7.3 Dynamic labeled 2-structures . . . . . . . . . . 450

6.7.4 Clans of a dynamic $\Delta^{\delta} 2$-structure $\ldots \ldots \ldots \ldots 454$

6.7 .5 Horizons . . . . . . . . . . . . . . . . 455

6.8 Dynamic $\ell 2$-structures with Variable Domains . . . . . . . . 457

6.8.1 Disjoint union of $\ell$-structures . . . . . . . . 457

6.8.2 Comparison with grammatical substitution . . . . . 458

6.8.3 Amalgamated union . . . . . . . . . . . . . . 459

6.9 Quotients and Plane Trees . . . . . . . . . . . . . . 460

6.9.1 Quotients of dynamic $\Delta^{\delta}$ 2-structures . . . . . . 460

6.9 .2 Plane trees . . . . . . . . . . . . . 461

6.10 Invariants . . . . . . . . . . . . . . . . . 469

6.10 .1 Introduction to invariants . . . . . . . . . 469

6.10 .2 Free invariants . . . . . . . . . . . . . . 470

6.10.3 Basic properties of free invariants . . . . . . . . . 4772

6.10 .4 Invariants on abelian groups . . . . . . . . . . . 473

6.10 .5 Clans and invariants . . . . . . . . . . . . 476

References . . . . . . . . . . . . . . . 476

7 Programmed Graph Replacement Systems $\quad 479$

(A. Schür) $\quad \mathbf{4 7 9}$

7.1 Introduction . . . . . . . . . . . . . . . . 481

7.1.1 Programmed Graph Replacement Systems in Practice . 481

7.1.2 Programmed Graph Replacement Systems in Theory . . 482

7.1.3 Contents of the Contribution . . . . . . . . . . . . 483

7.2 Logic-Based Structure Replacement Systems . . . . . . . . 484

7.2.1 Structure Schemata and Schema Consistent Structures . 485

7.2.2 Substructures with Additional Constraints . . . . . . . . 492

7.2.3 Schema Preserving Structure Replacement . . . . . . . 498

7.2 .4 Summary . . . . . . . . . . . . . . . . . 504

7.3 Programmed Structure Replacement Systems . . . . . . . . 505

7.3.1 Requirements for Rule Controlling Programs . . . . . 506

7.3 .2 Basic Control Flow Operators . . . . . . . . . . 507

7.3.3 Preliminary Definitions . . . . . . . . . . . 510

7.3.4 A Fixpoint Semantics for Transactions . . . . . . . . . 512

7.3 .5 Summary . . . . . . . . . . . . . . 518 
7.4 Context-sensitive Graph Replacement Systems - Overview . . . 519

7.4.1 Context-sensitive Graph Replacement Rules . . . . . . . 520

7.4.2 Embedding Rules and Path Expressions . . . . . . . . 523

7.4.3 Positive and Negative Application Conditions . . . . . . 526

7.4.4 Normal and Derived Attributes . . . . . . . . . 527

7.4 .5 Summary . . . . . . . . . . . . . . . . 530

7.5 Programmed Graph Replacement Systems - Overview . . . . . 530

7.5.1 Declarative Rule Regulation Mechanisms . . . . . . . 532

7.5.2 Programming with Imperative Control Structures . . . 534

7.5.3 Programming with Control Flow Graphs . . . . . . . 5 537

7.5 .4 Summary . . . . . . . . . . . . . . 540

References ....................... 541

$\begin{array}{ll}\text { Index } & 547\end{array}$ 